\title{
TOCOFEROIS E TOCOTRIENOIS EM ÓLEOS VEGETAIS E OVOS
}

\author{
Michele Guinazi \\ Faculdades Associadas do Espírito Santo, Faculdade de Saúde e Meio Ambiente. Rod. Serafim Derenzi, 3115, 29048-450 Vitória \\ - ES, Brasil \\ Regina Célia Rodrigues Miranda Milagres e Helena Maria Pinheiro-Sant’Ana* \\ Departamento de Nutrição e Saúde, Universidade Federal de Viçosa, Av. PH Rolfs, s/n, 36571-000 Viçosa - MG, Brasil \\ José Benício Paes Chaves \\ Departamento de Tecnologia de Alimentos, Universidade Federal de Viçosa, Av. PH Rolfs, s/n, 36571-000 Viçosa - MG, Brasil
}

Recebido em 7/11/08; aceito em 8/5/09; publicado na web em 6/10/09

\begin{abstract}
TOCOPHEROLS AND TOCOTRIENOLS IN VEGETABLE OILS AND EGGS. The distribution and content of vitamin E isomers was investigated in vegetable oils and raw and cooked egg yolk in commercial restaurants. The analysis of the eight vitamin $\mathrm{E}$ isomers was carried out by High Performance Liquid Chromatography (HPLC) with fluorescence detection. The tocopherol and tocotrienol composition of foods varied considerably. Tocopherols were detected in greater quantity and frequency. The $\alpha$-tocopherol predominated in egg yolks and olive oil while $\gamma$-tocopherol was found in high quantities in soybean and canola oils. Cooking did not cause major losses for most of the vitamin $\mathrm{E}$ isomers in egg yolks.
\end{abstract}

Keywords: vitamin E; edible oils; HPLC.

\section{INTRODUÇÃO}

O termo genérico "vitamina E" é utilizado para designar oito diferentes compostos, nomeados $\alpha-, \beta-, \gamma$ - e $\delta$ - (alfa, beta, gama e delta) tocoferois e tocotrienois. ${ }^{1-3}$

Tanto tocoferois como tocotrienois ocorrem em uma variedade de isômeros que diferem na estrutura de acordo com o número e a localização de grupos substituintes no anel cromanol. ${ }^{1,4}$ (Figura 1).

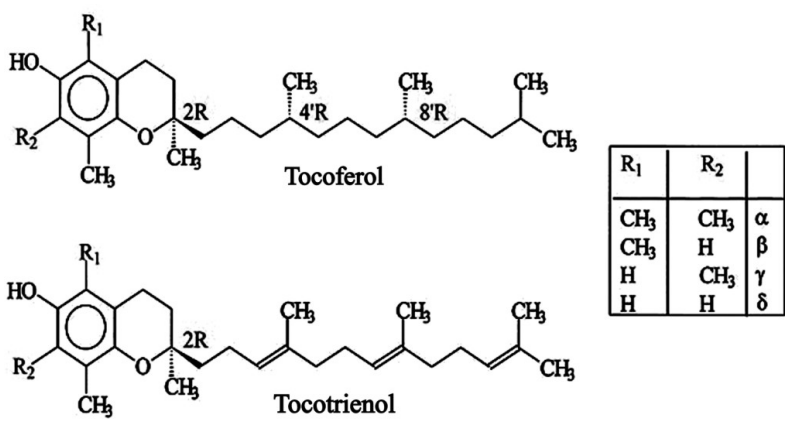

Figura 1. Estrutura química da vitamina E

A molécula tocol exibe isomerismo óptico atribuído aos três átomos de carbono assimétricos nas posições 2, 4' e 8', totalizando oito formas esteroisoméricas possíveis. O sistema $R S$ de configuração assimétrica é usado para especificar a quiralidade dos compostos da vitamina $\mathrm{E}$, de acordo com as normas da IUPAC. ${ }^{5}$

Tocoferois e tocotrienois são encontrados em proporções variáveis em plantas, sendo que as fontes principais são óleos vegetais, germe de trigo, sementes oleaginosas, vegetais folhosos verde-escuros e alimentos de origem animal, principalmente gema de ovo e fígado., ${ }^{4,6,7}$ Os isômeros mais abundantes nesses alimentos são o $\gamma$ - e o $\alpha$ - tocoferol. ${ }^{8}$ Atualmente, não se pode desconsiderar a utilização de alimentos enriquecidos e fortificados, como fontes ricas em vitamina $\mathrm{E}$.

\footnotetext{
*e-mail: helena.santana@ufv.br
}

A vitamina E tem sido extensivamente estudada em diversas áreas do conhecimento, uma vez que desempenha papeis especialmente importantes na reprodução normal e em mecanismos antioxidantes de tecidos animais e vegetais. ${ }^{9}$ Embora a deficiência não represente um problema de significância nutricional, a ingestão de vitamina E tem despertado interesse e preocupação principalmente nesta última década, uma vez que compõe juntamente com a vitamina $\mathrm{C}$, $\beta$-caroteno, selênio e flavonoides, o grupo denominado antioxidantes alimentares. Este grupo tem sido frequentemente associado à prevenção de doenças neurodegenerativas, aterosclerose, inflamação crônica, câncer e envelhecimento precoce..$^{10}$

Entre os compostos da vitamina E, o $\alpha$-tocoferol é apontado como sendo o mais potente em sua ação antioxidante. ${ }^{11}$ No entanto, alguns trabalhos indicam que outros isômeros como $\gamma$-e $\delta$-tocoferol são melhores antioxidantes. ${ }^{12-14}$ Essas controvérsias podem ocorrer devido a diferenças entre os métodos testados, os substratos utilizados, o nível de oxidação empregado e as metodologias empregadas para monitoramento da oxidação. ${ }^{13,14}$ Além disso, um número cada vez maior de estudos tem apontado os tocotrienois como sendo os compostos mais potentes na atividade antioxidante in vitro, mas pesquisas in vivo ainda devem ser melhor conduzidas. ${ }^{11,13,15}$

Os óleos vegetais comestíveis, além de possuírem altas concentrações de tocoferois e alguns tocotrienois, apresentam grande consumo em nível mundial, constituindo-se, portanto, nos alimentos de maior contribuição para a ingestão de vitamina E para a população. ${ }^{16} \mathrm{O}$ óleo de girassol parece ser o mais rico em $\alpha$-tocoferol, seguido pelo de algodão, palma, canola, amendoim, oliva, milho, soja e coco. O $\gamma$-tocoferol é o composto predominante em óleos de soja e de milho, sendo que o óleo de palma é o que apresenta maior teor de tocotrienois. ${ }^{17} \mathrm{O}$ óleo de soja, devido ao grande consumo em nível mundial, é o principal contribuinte para a ingestão de vitamina E pela população. ${ }^{16}$

O conteúdo de tocoferois e tocotrienois em óleos vegetais é diretamente relacionado com o tipo de processamento aplicado. Assim, óleos refinados contêm um teor vitamínico reduzido em até $80 \%$, de acordo com as condições empregadas. ${ }^{18}$ Além disso, perdas podem acontecer depois de embalados, de acordo com as formas de estocagem, pela exposição à luz, oxigênio, altas temperaturas, entre outros fatores. ${ }^{19-21}$ 
Com relação aos alimentos de origem animal, os ovos apresentam os maiores conteúdos em vitamina $\mathrm{E}$, no entanto, o efeito do enriquecimento na alimentação de aves, comum em muitos países, não pode ser esquecido. ${ }^{22,23}$

No Brasil, dados do IBGE ${ }^{24}$ apontam para um consumo per capita anual de 4,3 $\mathrm{kg}$ de ovos e de 7,5 kg de óleos, demonstrando que estes alimentos são amplamente consumidos pela população.

Diversas técnicas têm sido aplicadas para separação, purificação e quantificação de tocoferois e tocotrienois nas mais variadas matrizes orgânicas e podem ser utilizadas de acordo com os objetivos da análise e da natureza da amostra. ${ }^{25,26}$ Entre as técnicas empregadas, podem-se citar as colorimétricas, cromatografia em camada delgada, a cromatografia gasosa, a cromatografia líquida de alta eficiência (CLAE), além de algumas ainda em desenvolvimento, como a eletroforese capilar. ${ }^{26-28}$

Desde que foi utilizada para a análise de antioxidantes lipídicos, a CLAE tem sido extensivamente aplicada e é preferida pela grande maioria dos pesquisadores da área. ${ }^{29}$ Rios e Penteado ${ }^{30}$ demonstraram em seu estudo em alhos irradiados que a CLAE de fase normal com sistema de eluição isocrática é adequada para resolução do $\alpha$-tocoferol.

Poucos trabalhos têm pesquisado a composição completa dos isômeros da vitamina $\mathrm{E}$ em alimentos, sendo que a maioria tem relatado apenas o conteúdo do $\alpha$-tocoferol. Além disso, as Tabelas Brasileiras de Composição de Alimentos ainda não contemplam os diferentes isômeros da vitamina $\mathrm{E}$, o que torna importante as pesquisas envolvendo a composição completa em alimentos brasileiros.

Com base nessas observações, o objetivo desse estudo foi investigar a distribuição e a concentração dos oito isômeros da vitamina E em óleos vegetais comestíveis e ovos preparados em restaurantes comerciais, por meio da CLAE.

\section{PARTE EXPERIMENTAL}

\section{Amostras}

Utilizaram-se óleos vegetais refinados (soja, canola e oliva extra-virgem) adquiridos no comércio local e gemas de ovo (cruas e cozidas) preparadas em restaurantes comerciais de Viçosa, MG. É importante ressaltar que se optou pela investigação somente da gema em decorrência do conteúdo de vitamina $\mathrm{E}$ ser superior a do ovo inteiro, cerca de duas vezes e meia. ${ }^{17}$

\section{Reagentes e outros materiais}

Para o preparo das amostras utilizaram-se os seguintes reagentes com grau de pureza analítica (P.A): acetato de etila, hexano, isopropanol, e butil-hidroxitolueno - BHT (Synth, Brasil) e sulfato de sódio anidro (Vetec, Brasil).

Os padrões vitamínicos utilizados foram os isômeros $\alpha-, \beta-, \gamma$ - e $\delta$ - tocoferol e tocotrienol (Calbiochem ${ }^{\circledR}$, EMD Biosciences, Inc., EUA). As fases móveis foram preparadas utilizando reagentes de grau cromatográfico: hexano e isopropanol (Mallinckrodt, EUA) e ácido acético (Merck, Brasil). Para a filtração das amostras utilizou-se papel de filtro qualitativo, filtração média (J. Prolab, Brasil); seringas descartáveis esterilizadas de $3 \mathrm{~mL}$ (Rymco, Colômbia). Antes das análises cromatográficas as amostras foram novamente filtradas em unidades filtrantes HV Millex, em polietileno, 0,45 $\mu \mathrm{m}$ de porosidade (Millipore, Brasil).

\section{Equipamentos}

Para extração dos isômeros da vitamina E nas amostras, utilizouse: microtriturador (modelo MA 102, Marconi); bomba de vácuo (modelo CA Fanen); evaporador rotativo (Q-344.1, Quimis). As fases móveis foram degaseificadas em vibrador ultrassônico (T-14 Odontobrás). Para obtenção dos espectros de absorção e avaliação da pureza dos padrões vitamínicos foi utilizado espectrofotômetro (UV 1601, Shimadzu). Para a determinação de sólidos totais, utilizou-se estufa de circulação forçada de ar (Fanen 320-SE) e balança analítica com 5 casas decimais (Ohaus Explorer).

Para as análises cromatográficas utilizou-se sistema de cromatografia líquida de alta eficiência (Shimadzu, modelo SCL 10AT VP) composto de bomba de alta pressão (modelo LC-10AT VP); injetor automático com loop de $50 \mu \mathrm{L}$ (modelo SIL-10AF); coluna LiChrosorb (Si60 Phenomenex 250 x 4 mm, $5 \mu \mathrm{m}$ ); detector de fluorescência (modelo RF-10A XL); software "Multi System" (modelo Class VP 6.1).

\section{Restaurantes colaboradores e coleta das amostras}

Nove restaurantes comerciais localizados na cidade de Viçosa, MG, Brasil foram pré-selecionados e contatados. Considerando os hábitos alimentares da região, a semelhança dos procedimentos operacionais e a periodicidade de entrada dos alimentos selecionados no cardápio, dois restaurantes foram escolhidos e convidados a colaborar com o estudo.

Os ovos foram preparados nos restaurantes selecionados de acordo com os métodos de rotina utilizados em cada local, possibilitando a análise do conteúdo vitamínico em condições reais de preparação e consumo.

Uma vez preparados, foram coletadas amostras dos alimentos, embaladas em sacos plásticos, identificadas e levadas ao laboratório, onde foram armazenadas em geladeira sob temperatura de 0 a $5{ }^{\circ} \mathrm{C}$, até o momento da extração e análise.

Para a análise do conteúdo vitamínico em óleos vegetais, os mesmos foram adquiridos no comércio local e as determinações conduzidas em três repetições. Para uma melhor representatividade da análise, tomou-se o cuidado de adquirir unidades dos óleos que fossem da mesma marca, porém, provenientes de diferentes lotes de fabricação.

\section{Preparação das amostras para análise}

Utilizou-se método de extração direta com solventes para análise dos oito isômeros da vitamina $\mathrm{E}$ em gema de ovos, de acordo com Guinazi. ${ }^{31}$ Para tanto, pesou-se em torno de $10 \mathrm{~g}$ da amostra e, em seguida, adicionou-se $4 \mathrm{~mL}$ de água deionizada aquecida (aproximadamente $80^{\circ} \mathrm{C}$ ) e cerca de $5 \mathrm{~g}$ de sulfato de sódio anidro. Acrescentouse $10 \mathrm{~mL}$ de isopropanol e $1 \mathrm{~mL}$ de hexano contendo $0,05 \%$ de BHT. Após a adição de $25 \mathrm{~mL}$ de mistura solvente (hexano:acetato de etila, $85: 15, \mathrm{v} / \mathrm{v})$, as amostras foram trituradas e filtradas a vácuo. Lavou-se o resíduo retido pela filtração com $15 \mathrm{~mL}$ de mistura solvente. Em seguida, transferiu-se o mesmo para o tubo de trituração e a etapa de extração foi repetida adicionando-se $5 \mathrm{~mL}$ de isopropanol e $30 \mathrm{~mL}$ de mistura solvente, com posterior homogeneização e filtração a vácuo. O resíduo foi novamente lavado com $5 \mathrm{~mL}$ de mistura solvente e evaporado em rotavapor, utilizando temperatura de $70^{\circ} \mathrm{C}$. As amostras foram dissolvidas em mistura solvente, sendo o volume completado com a mesma mistura para $25 \mathrm{~mL}$, utilizando balões volumétricos. Após a completa homogeneização, tomou-se uma alíquota de $5 \mathrm{~mL}$ e evaporou-se em nitrogênio gás, armazenando-se em vidros âmbar hermeticamente vedados em temperatura de -5 a $0{ }^{\circ} \mathrm{C}$, até o momento da análise cromatográfica.

Todas as operações foram efetuadas ao abrigo da luz natural e fluorescente, utilizando-se vidraria de cor âmbar ou proteção com papel alumínio.

Para as análises dos óleos vegetais não houve necessidade de realizar a etapa de extração. 


\section{Determinação dos tocoferois e tocotrienois}

As análises dos tocoferois e tocotrienois foram conduzidas por CLAE, utilizando-se as condições cromatográficas propostas por Guinazi: ${ }^{31}$ coluna LiChrosorb Si60, $5 \mu \mathrm{m} ; 250$ x 4 mm; detecção por fluorescência ( $290 \mathrm{~nm}$ de excitação e $330 \mathrm{~nm}$ de emissão); fase móvel composta de hexano/isopropanol/ácido acético (99,33:0,6:0,07), com

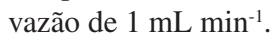

Após extração, as alíquotas das amostras secas em nitrogênio foram redissolvidas em $2 \mathrm{~mL}$ de hexano e filtradas através de unidades filtrantes com porosidade de $0,45 \mu \mathrm{m}$. As análises foram realizadas injetando-se dois volumes diferentes para cada amostra, a fim de se obter a detecção de todos os compostos em quantidades apropriadas para a identificação e quantificação. Dessa forma, injetou-se $5 \mu \mathrm{L}$ dos extratos para análise do $\alpha$-tocoferol e $50 \mu \mathrm{L}$ para detecção dos demais isômeros que ocorrem em menor concentração.

A injeção dos óleos na coluna cromatográfica foi realizada após diluição de $0,1 \mathrm{~g}$ da amostra em $10 \mathrm{~mL}$ de hexano, seguida por filtração em unidades filtrantes com porosidade de 0,45 $\mu \mathrm{m}$ (adaptado de Syväoja et al., ${ }^{32}$ Dionise et al. ${ }^{33}$ ).

Visto que os alimentos estudados possuem natureza oleosa, sua injeção para análise tende a sujar a coluna cromatográfica e consequentemente alterar os tempos de retenção dos analitos. Assim, para minimizar esse problema, tomou-se o cuidado de lavar a coluna diariamente com hexano após as corridas cromatográficas.

\section{Curvas analíticas, identificação e quantificação dos tocoferois e} tocotrienois

Isômeros $\alpha$-, $\beta$-, $\gamma$ - e $\delta$-tocoferol e tocotrienol foram utilizados para o preparo das soluções padrão. Soluções estoques de cada um dos oito compostos foram preparadas a partir da pesagem de $5 \mathrm{mg}$ de cada padrão, que foram dissolvidos em hexano contendo $0,01 \%$ de BHT e o volume completado para $100 \mathrm{~mL}$, obtendo-se concentrações de $50 \mu \mathrm{g} \mathrm{mL} \mathrm{L}^{-1}$.

A concentração exata das soluções padrão de cada isômero foi determinada espectrofotometricamente. ${ }^{34}$ A pureza encontrada para os diferentes isômeros variou entre 89,7 e $97,6 \%$. As soluções estoque foram mantidas em vidro âmbar a $4{ }^{\circ} \mathrm{C}$ e a concentração das mesmas foi verificada mensalmente por espectrofotometria.

A identificação dos oito isômeros da vitamina $\mathrm{E}$ nas amostras foi realizada comparando-se os tempos de retenção obtidos para os padrões e para as amostras, analisados sob as mesmas condições e por cocromatografia. Para tanto, uma solução padrão contendo uma mistura dos oito isômeros foi injetada diariamente. Além disso, avaliou-se a cada análise, a sequência esperada para cada um dos isômeros de acordo com a literatura. ${ }^{25}$

A quantificação dos compostos foi feita utilizando curvas analíticas para cada um dos oito isômeros. A construção das curvas foi feita através de injeção, em triplicata, de cinco concentrações crescentes de soluções padrão de cada isômero (entre 0,5 e $5 \mu \mathrm{g} \mathrm{mL}^{-1}$ ). Desse modo, foi feita uma correlação linear entre as áreas dos picos e as concentrações injetadas de cada composto. A equação de regressão linear obtida para cada isômero foi utilizada para quantificar o conteúdo dos tocoferois e tocotrienois nas amostras de alimentos estudadas.

\section{Delineamento experimental e análise estatística dos dados}

Utilizou-se um delineamento experimental inteiramente casualizado em esquema fatorial, cujos fatores foram dois restaurantes, dois tipos de alimentos (óleos e ovos) e três repetições.

Para a comparação de médias do teor dos compostos nos alimentos entre os dois restaurantes, aplicou-se o teste de t Student, a nível de 5\% de probabilidade $(\alpha=5 \%)$. Para a comparação de médias dos valores dos compostos entre os alimentos, aplicou-se o teste de amplitude múltiplas de Duncan $(\alpha=5 \%)$. Todas as análises estatísticas foram conduzidas utilizando-se o software SAS (Statistical Analisys System).

\section{RESULTADOS E DISCUSSÃO}

A faixa de linearidade obtida para os oito isômeros da vitamina E foi ampla (entre 2,5 e $135 \mathrm{ng}$ ), o que garantiu a obtenção de dados confiáveis dentro das faixas analisadas. O coeficiente de determinação $\left(\mathrm{R}^{2}\right)$ foi maior que 0,995 para os oito isômeros.

A recuperação dos padrões dos oito isômeros adicionados às amostras dos alimentos estudados variou entre 91,3 e 99,5\% e indica que a metodologia utilizada para extração e análise dos compostos da vitamina E em óleos vegetais e ovos foi utilizada com segurança.

\section{Análise qualitativa da vitamina $\mathbf{E}$}

A Figura 2 apresenta um perfil cromatográfico típico obtido para a mistura das soluções-padrão dos oito isômeros da vitamina E. A eluição dos compostos seguiu a ordem característica e já bem estabelecida pela literatura para análises utilizando sistema de fase normal, a saber: $\alpha$-tocoferol, $\alpha$-tocotrienol, $\beta$ - tocoferol, $\gamma$ - tocoferol, $\beta$ - tocotrienol, $\gamma$ - tocotrienol, $\delta$ - tocoferol, $\delta$ - tocotrienol. ${ }^{25}$

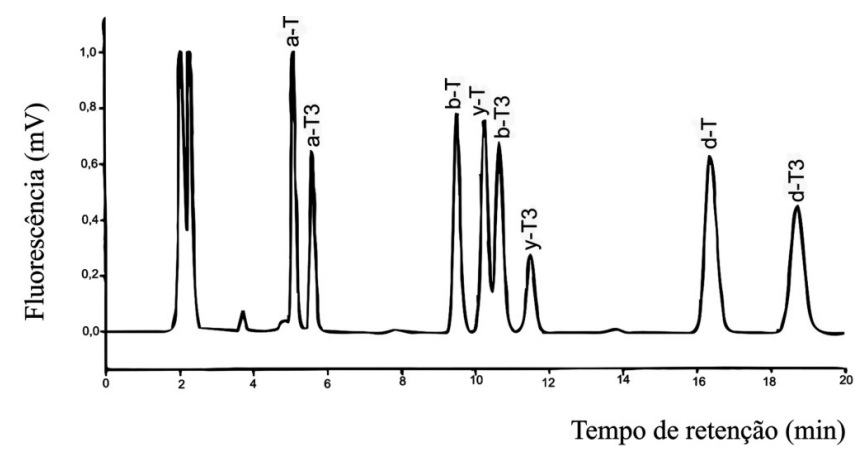

Figura 2. Cromatograma da mistura de padrões de tocoferois $(T)$ e tocotrienois $(T 3) .(a)=$ alfa $;(b)=$ beta $;(y)=$ gama $;(d)=$ delta. Condições cromatográficas encontram-se na parte experimental

A Figura 3 mostra cromatogramas típicos das amostras de gema de ovo cru e cozida analisadas neste trabalho. Verifica-se que o método de preparo das amostras permitiu uma excelente resolução e quantificação dos compostos de interesse.

Todos os compostos da vitamina E ocorreram nas amostras, com exceção do $\delta$ - tocotrienol. $O$ isômero $\alpha$-tocoferol foi predominante, sendo que os demais compostos ocorreram em quantidades menos expressivas.

Os dados qualitativos são coincidentes com as informações encontradas na literatura que indicam que o $\alpha$-tocoferol é o composto predominante em ovos, enquanto que isômeros como $\beta$-tocoferol, $\beta$-tocotrienol e $\gamma$-tocotrienol ocorrem em menor quantidade. ${ }^{35}$

Os cromatogramas obtidos para os óleos (Figura 4) mostram que a composição dos isômeros variou bastante de acordo com a espécie vegetal da qual o óleo é originado, o que está de acordo com informações de Cert et al.. ${ }^{36}$ Apesar da identificação ter sido comprovada pela injeção da mistura de padrões no mesmo dia (tempos de retenção dos analitos identificados nos óleos vegetais coincidentes aos dos padrões), o tempo de retenção dos isômeros nos óleos vegetais mostrou-se diferente daqueles observados nos demais alimentos, devido 

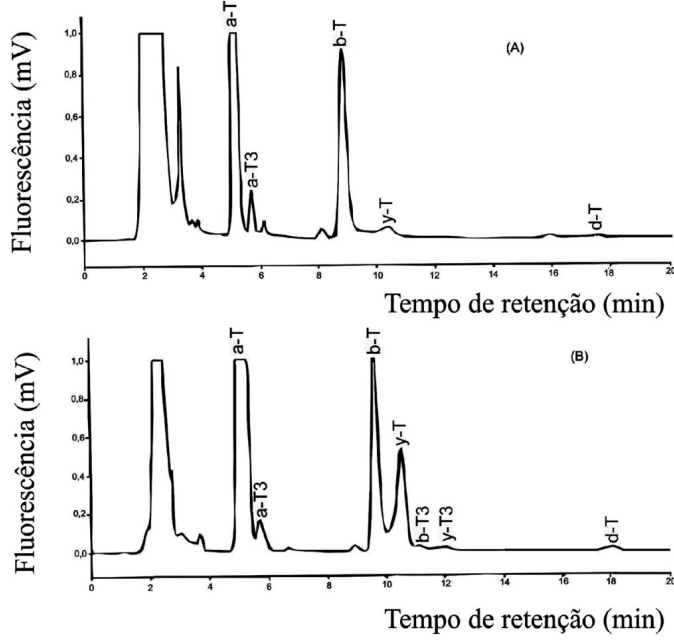

Figura 3. Cromatograma de tocoferois (T) e tocotrienois (T3) em amostras de gema de ovo crua $(A)$ e gema de ovo cozida $(B) .(a)=a l f a ;(b)=b e t a ;(y)=$ gama; $(d)=$ delta. Condições cromatográficas encontram-se na parte experimental

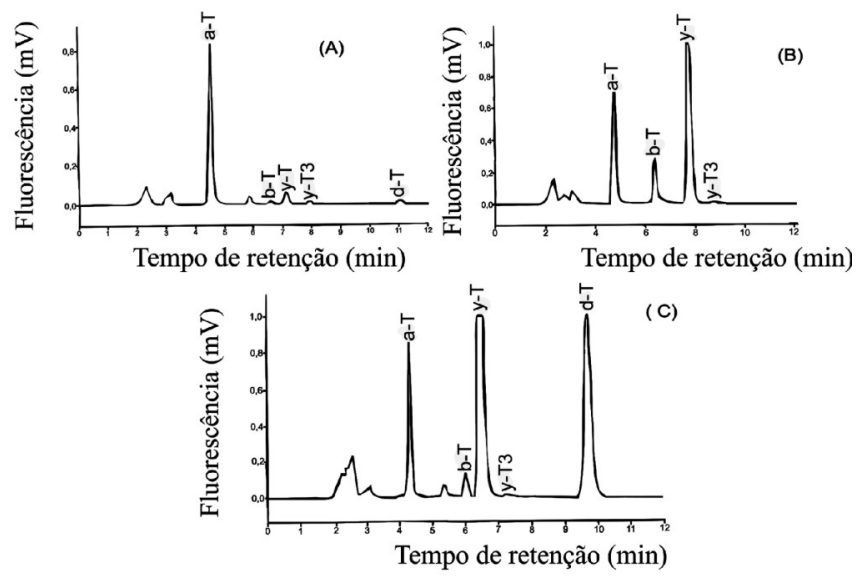

Figura 4. Cromatograma de tocoferois (T) e tocotrienois (T3) em amostras de óleo de oliva $(A)$, óleo de canola $(B)$ e óleo de soja $(C) .(a)=$ alfa; $(b)=$ beta; $(y)=$ gama $;(d)=$ delta OU (tocoferol e tocotrienol). Condições cromatográficas encontram-se na parte experimental possivelmente à variabilidade encontrada no sistema de fase normal. Assim, além dos cuidados acima, a identificação foi complementada pela análise da sequência esperada para os diferentes isômeros.

O perfil cromatográfico do óleo de oliva mostra que o $\alpha$-tocoferol foi o composto principal, enquanto os demais ocorreram em menores quantidades relativas, sendo que em estudos anteriores nem chegaram a ser detectados. ${ }^{17,32,33}$

Os óleos de canola e de soja apresentaram um perfil cromatográfico semelhante, onde o $\gamma$-tocoferol foi o composto predominante. $\mathrm{O} \alpha$ - e o $\beta$-tocoferol ocorreram em menores concentrações. O óleo de soja apresentou o $\delta$-tocoferol, composto que ocorre com menor frequência em outros alimentos.

\section{Análises cromatográficas quantitativas}

A concentração de tocoferois e tocotrienois em amostras de ovos analisados crus e após preparação, nos dois restaurantes selecionados, está apresentada na Tabela 1 .

A concentração dos oito isômeros da vitamina $\mathrm{E}$ em gemas de ovos cruas não diferiu estatisticamente em relação aos dois restaurantes pesquisados. Entretanto, em gemas de ovos cozidas no restaurante 2 os teores de $\alpha$-tocoferol e de $\gamma$-tocoferol foram significativamente superiores $(\mathrm{p}>0,05)$. Esses achados sugerem que a estabilidade dos isômeros foi dependente da diferente forma de preparação utilizada pelos restaurantes.

Observa-se que a composição de tocoferois e tocotrienois variou amplamente neste estudo, mas, de forma geral, os isômeros tocoferois foram encontrados em quantidades superiores aos tocotrienois.

O cozimento de ovos reduziu o conteúdo de alguns compostos, podendo indicar uma possível degradação dos isômeros devido à exposição do alimento a altas temperaturas e tempo prolongado de cocção.

Syväoja et al. ${ }^{22}$ encontraram teores de $55,0 \mathrm{mg} \mathrm{kg}^{-1}$ de $\alpha$-tocoferol em gema crua, valor muito superior ao relatado por outros estudos. Teores de $\alpha$-tocoferol variando de 5,0 a $27,5 \mathrm{mg} \mathrm{kg}^{-1}$ podem ser considerados próximos ao encontrado neste estudo, onde as concentrações variaram entre 8,3 a $11,3 \mathrm{mg} \mathrm{kg}^{-1}$ na gema crua. ${ }^{9,22}$

Os teores de vitamina E obtidos para óleos de soja, de canola e óleo de oliva extra-virgem são apresentados na Tabela 2.

Observa-se que, de modo geral, os óleos avaliados apresentaram elevadas quantidades dos isômeros de tocoferois, sendo que concentrações mais reduzidas de $\gamma$-tocotrienol foram detectadas. Diversos

Tabela 1. Conteúdo de tocoferois e tocotrienois (Média \pm Desvio Padrão; em mg kg-1 de matéria fresca) em ovos crus e cozidos, preparados em restaurantes comerciais

\begin{tabular}{|c|c|c|c|c|}
\hline \multirow{2}{*}{ Isômero } & \multicolumn{2}{|c|}{ Ovo (Gema) Cru } & \multicolumn{2}{|c|}{ Ovo (Gema) Cozido } \\
\hline & $\mathrm{R} 1 *$ & $\mathrm{R} 2 *$ & $\mathrm{R} 1 *$ & $\mathrm{R} 2 *$ \\
\hline$\alpha$-tocoferol & $8,26^{\mathrm{a}} \pm 0,95$ & $11,27^{\mathrm{a}} \pm 1,79$ & $7,17^{\mathrm{b}} \pm 0,50$ & $10,14^{a} \pm 0,62$ \\
\hline$\beta$ - tocoferol & $0,09 \pm 0,02$ & nd & $\operatorname{tr}$ & nd \\
\hline$\gamma$ - tocoferol & $\operatorname{tr}$ & nd & $2,04^{\mathrm{b}} \pm 0,45$ & $5,47^{\mathrm{a}} \pm 0,44$ \\
\hline$\delta$ - tocoferol & $\operatorname{tr}$ & $0,04 \pm 0,00$ & $\operatorname{tr}$ & $\operatorname{tr}$ \\
\hline$\alpha$-tocotrienol & $0,86^{\mathrm{a}} \pm 0,08$ & $0,89^{\mathrm{a}} \pm 0,02$ & $0,74^{\mathrm{a}} \pm 0,13$ & $0,82^{\mathrm{a}} \pm 0,07$ \\
\hline$\beta$ - tocotrienol & $\operatorname{tr}$ & nd & $1,72 \pm 2,22$ & nd \\
\hline$\gamma$ - tocotrienol & nd & $0,17 \pm 0,12$ & $\operatorname{tr}$ & $0,15 \pm 0,08$ \\
\hline$\delta$ - tocotrienol & nd & nd & nd & nd \\
\hline
\end{tabular}

${ }^{a}$ Médias ( $\mathrm{n}=3$ ) seguidas da mesma letra na linha, por preparação, não diferem entre si pelo Teste de $t$ Student $(\alpha=5 \%)$.

* R1 e R2 = Restaurante 1 e 2; tr: abaixo do limite de quantificação do método; nd: isômero não detectado (ausência de pico do isômero nos cromatogramas). 
Tabela 2. Conteúdo de tocoferois e tocotrienois (Média \pm Desvio Padrão em $\mathrm{mg} \mathrm{kg}^{-1}$ ) em óleos vegetais comestíveis

\begin{tabular}{lccc}
\hline Isômero & \multicolumn{1}{c}{} & \\
& Oliva & Óleos Vegetais & Soja \\
\hline$\alpha-\mathrm{T}$ & $140,47^{\mathrm{b}} \pm 16,81$ & $183,93^{\mathrm{a}} \pm 9,64$ & $121,40^{\mathrm{b}} \pm 16,24$ \\
$\beta-\mathrm{T}$ & $4,74^{\mathrm{c}} \pm 0,87$ & $51,58^{\mathrm{a}} \pm 14,29$ & $28,15^{\mathrm{b}} \pm 4,41$ \\
$\gamma-\mathrm{T}$ & $15,15^{\mathrm{c}} \pm 1,57$ & $390,24^{\mathrm{b}} \pm 89,29$ & $642,73^{\mathrm{a}} \pm 39,82$ \\
$\delta$-T & $3,69^{\mathrm{b}} \pm 1,90$ & $11,90^{\mathrm{b}} \pm 0,31$ & $225,72^{\mathrm{a}} \pm 12,20$ \\
$\alpha-\mathrm{T} 3$ & nd & nd & nd \\
$\beta-\mathrm{T} 3$ & nd & nd & nd \\
$\gamma$-T3 & $12,28^{\mathrm{a}} \pm 0,88$ & $11,42^{\mathrm{a}} \pm 2,18$ & $8,10^{\mathrm{a}} \pm 1,41$ \\
$\delta$-T3 & nd & nd & nd \\
\hline
\end{tabular}

Médias seguidas (n=3: três amostras diferentes de óleos vegetais, obtidas de diferentes lotes, caracterizando 3 repetições) de pelo menos uma letra na linha não diferem entre si $(\alpha=5 \%)$, pelo teste de Duncan. nd: isômero não detectado (ausência de pico do isômero nos cromatogramas).

estudos mostram que os tocotrienois não são compostos de ocorrência comum em óleos de soja e canola, e são apontados como os melhores marcadores para avaliação da pureza de óleo de oliva. ${ }^{16,32}$

Com relação aos teores de $\alpha$-tocoferol, o óleo de canola apresentou um conteúdo significantemente maior que o encontrado em óleos de soja e oliva. No entanto, considerando a utilização desses óleos, pode-se apontar o óleo de soja como uma das principais fontes de vitamina $\mathrm{E}$ para as populações ocidentais, uma vez que é o óleo comestível mais difundido e utilizado para preparação de alimentos e como matéria-prima de outros produtos alimentícios, como margarina e maionese. ${ }^{16}$

Exceto para o óleo de oliva, o $\gamma$-tocoferol foi o composto encontrado em maior concentração nos óleos analisados, especialmente no óleo de soja. ${ }^{8} 16$ A riqueza em $\gamma$-tocoferol e a grande utilização do óleo de soja o tornava a principal fonte de equivalentes de $\alpha$-tocoferol, antes da revisão das recomendações pelo Instituto de Medicina dos Estados Unidos. Após a indicação de uso da Ingestão Diária Recomendada (IDR), a grande concentração do composto foi totalmente desprezada para aproveitamento nutricional em humanos, mas seu papel como um dos principais colaboradores no sistema antioxidante de vegetais não pode ser esquecido. ${ }^{8}$

Além de concentrações moderadas de $\beta$-tocoferol, é interessante destacar que os óleos vegetais apresentaram quantidades apreciáveis de $\delta$-tocoferol, um dos mais potentes antioxidantes em alimentos, $o$ que indica a forte correlação antioxidante existente entre a vitamina E e a presença de ácidos graxos insaturados. ${ }^{9}$ Neste estudo, o óleo de soja apresentou um conteúdo em $\delta$-tocoferol significantemente maior que os demais óleos analisados.

Estudos realizados na Finlândia, Estados Unidos e Espanha informam teores em óleo de soja que variam entre 12,0 e 119,0 mg kg-1 para $\alpha$-tocoferol; 14 e $699 \mathrm{mg} \mathrm{kg}^{-1}$ para $\gamma$-tocoferol e 166,0 e $248,0 \mathrm{mg} \mathrm{kg}^{-1}$ para $\delta$-tocoferol. ${ }^{17,32,37,38}$ Comparando os valores encontrados para estes compostos nas amostras de óleo de soja avaliadas neste estudo (121,4 $\mathrm{mg} \mathrm{kg}^{-1} \mathrm{de} \alpha$ - tocoferol; 642,7 $\mathrm{mg} \mathrm{kg}^{-1}$ de $\gamma$ - tocoferol e $225,7 \mathrm{mg} \mathrm{kg}^{-1}$ de $\delta$-tocoferol), observa-se que os resultados estão dentro da faixa superior mencionada acima, e indicam que o óleo de soja brasileiro é uma fonte importante de vitamina E. Os resultados obtidos para óleo de oliva $(140,4$ $\mathrm{mg} \mathrm{kg}^{-1}$ de $\alpha$-tocoferol) também são condizentes com os valores encontrados na literatura (107,0 a $\left.235,0 \mathrm{mg} \mathrm{kg}^{-1}\right)$, embora a maior parte dos estudos não tenham detectado outros isômeros além do $\alpha$-tocoferol em suas análises. , $^{217,32,33}$

Os valores encontrados para $\alpha$-tocoferol $\left(183,9 \mathrm{mg} \mathrm{kg}^{-1}\right) \mathrm{e}$ $\gamma$-tocoferol (390,2 $\left.\mathrm{mg} \mathrm{kg}^{-1}\right)$ em óleo de canola mostraram-se condizentes com teores indicados na literatura (132,0 a 410,0 mg kg-1 e
42,0 a 287,0 $\mathrm{mg} \mathrm{kg}^{-1}$ de $\alpha$ - e $\gamma$-tocoferol, respectivamente), apesar da grande variação no teor dos compostos que parece existir nesse tipo de óleo., ${ }^{437-39}$

Outros óleos vegetais encontrados na natureza foram pesquisados por diferentes autores quanto aos teores de tocoferóis. Em Viena, na Áustria, estudos revelaram teores mais elevados do que os encontrados em nosso estudo para os isômeros $\alpha$ - tocoferol (1920,0 $\mathrm{mg} \mathrm{kg}^{-1}$ ) e $\gamma$-tocoferol $\left(720,0 \mathrm{mg} \mathrm{kg}^{-1}\right)$ em óleos vegetais. ${ }^{40}$ Vallilo et al..$^{41}$ ao pesquisarem óleos das sementes de Platymiscium floribundum Vog. (sacambu) verificaram teores superiores de $\alpha$ - e $\gamma$-tocoferol (428,0 e $882,0 \mathrm{mg} \mathrm{kg}^{-1}$, respectivamente). Entretanto, quantidades inferiores desses isômeros foram obtidas por Takemoto et al. ${ }^{42}$ em óleos de semente de baru (50,0 e 43,0 $\mathrm{mg} \mathrm{kg}^{-1}$, respectivamente ).

É importante ressaltar que as análises em nosso estudo foram conduzidas com óleos refinados, a fim de estimar o consumo real pela população. No entanto, a literatura é unânime em afirmar que grandes perdas de vitamina E ocorrem durante a etapa de extração e refinamento do óleo, onde altas temperaturas são utilizadas, bem como na estocagem do produto, por processos de oxidação iniciados pela luz e efeito do oxigênio. ${ }^{9,12,39,42}$ Syavoja et al.,${ }^{17}$ estudando o efeito do refinamento sobre o teor de vitamina $\mathrm{E}$ em óleos vegetais, observaram perdas de 10 a $33 \%$ de $\alpha$-tocoferol, 20 a $33 \%$ dos demais tocoferois e aproximadamente $50 \%$ de tocotrienois.

Considerando a contribuição dos óleos vegetais na ingestão diária de vitamina E para a população brasileira, é interessante fazer um paralelo entre os resultados aqui obtidos com a quantidade recomendada de vitamina $\mathrm{E}$ a ser consumida. Dessa forma, a contribuição do consumo de óleos analisados para a ingestão diária recomendada de vitamina E é mostrada na Figura 5.

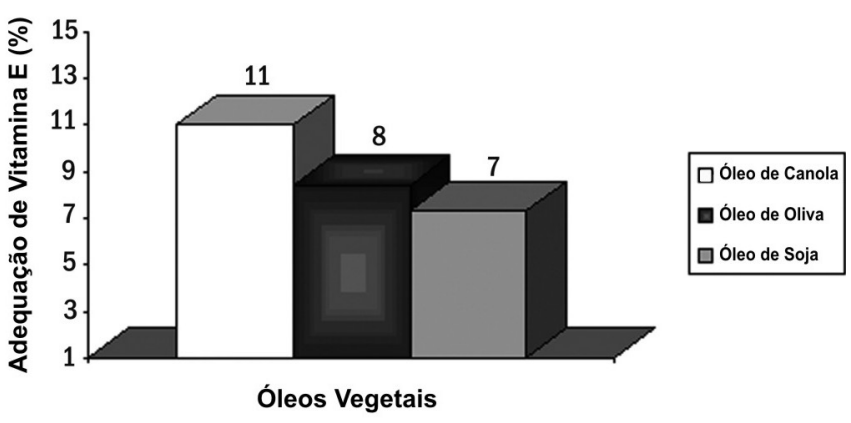

Figura 5. Percentual de adequação quanto à ingestão dietética de referência de vitamina $E$ (considerando os teores de $\alpha$-tocoferol) a partir do consumo de uma porção $(9 \mathrm{~g})$ de óleos vegetais comestíveis diariamente 
Para realizar essa comparação, tomou-se como base a recomendação diária de vitamina $\mathrm{E}$ para um indivíduo adulto, além de porções de óleos que são indicadas pela Pirâmide de Alimentos, um guia alimentar já bem estabelecido. A adequação pôde ser calculada, portanto, a partir da recomendação de $15 \mathrm{mg}$ de $\alpha$-tocoferol/dia e a ingestão de uma porção de óleo (9 g) ao dia. ${ }^{43,44}$

Observa-se que a ingestão de óleos de canola, oliva e soja é importante para a ingestão diária de vitamina E, pois, em pequenas quantidades, fornecem uma adequação relevante. A ingestão de uma porção de óleo de canola, por exemplo, corresponde a $11 \%$ da quantidade diária recomendada de vitamina E. No entanto, é interessante ressaltar que cada vez mais os especialistas e comitês de alimentação e nutrição têm recomendado o uso moderado de óleos e gorduras e o maior consumo de frutas e hortaliças, para a promoção e manutenção da saúde. ${ }^{17,45}$

Portanto, a avaliação dos isômeros da vitamina $\mathrm{E}$ em alimentos consumidos pela população brasileira, como ovos e óleo, fornece dados importantes sobre o seu valor nutritivo, além de contribuir para a caracterização do potencial antioxidante desses alimentos.

A composição de vitamina $\mathrm{E}$ mostrou-se bastante variável, o que ressalta a importância de estudos de composição de alimentos brasileiros, produzidos e manipulados sob condições locais, que permitam refletir com maior veracidade o consumo dos nutrientes pela população.

\section{CONCLUSÕES}

Os óleos vegetais analisados neste estudo mostraram ser ricos em $\alpha$-tocoferol, especialmente o óleo de oliva extra-virgem, contribuindo de forma relevante para a adequação diária de vitamina E, se consumidos em porções adequadas. Os óleos de soja e canola apresentaram teores elevados de $\gamma$ - e $\delta$-tocoferol e de $\delta$-tocotrienol, o que reforça a sua importância na dieta como fontes de antioxidantes naturais. O cozimento de ovos nas condições avaliadas não ocasionou perdas importantes para a maioria dos isômeros da vitamina $\mathrm{E}$. Os dados deste estudo representam uma grande contribuição para a caracterização nutricional dos alimentos avaliados, uma vez que trabalhos desta natureza são escassos no Brasil.

\section{REFERENCIAS}

1. American Institute of Nutrition - AIN; J. Nutr. 1979, 8.

2. Sen, C. K.; Khanna, S.; Roy, S.; Life Sci. 2006, 78, 2088.

3. Chun, J.; Lee, J.; Ye, L.; Exler, J.; Eitenmiller, R. R.; J. Food Composit. Anal. 2006, 19, 196

4. Sheppard, A. J.; Pennington, J. A. T.; Weihrauch J. L. Em Vitamin E in health and disease; Packer, L.; Fuchs, J., eds.; Marcel Dekker: New York, 1992, cap. 1.

5. Ball, G. F. M.; Vitamin E Bioavailability and analysis of vitamins in foods, Chapman \& Hall: London, 1998, cap. 5.

6. Chen, J. Y.; Latshaw, J. D.; Lee, H. O.; Min, D. B.; J. Food Sci. 1998, 6 , 919.

7. Sridhar, R.; Lakshminarayana, G.; J. Agric. Food Chem. 1993, 41, 61.

8. Jiang, Q.; Christen, S.; Shigenaga, M. K.; Ames, B. N.; Am. J. Clin. Nutr. 2001, 74, 714 .

9. Azzi, A.; Stocker, A.; Prog. Lipid Res. 2000, 39, 231

10. Traber, M. G.; Am. J. Clin. Nutr. 2001, 73, 997; Bieri, J. G.; Am. J. Clin. Nutr. 2002, 75, 781; Bron, D.; Amis, R.; J. Vitam. Nut. Res. 2002, 72, 32

11. Yoshida, Y.; Niki, E.; Noguchi, N.; Chem. Phys. Lipids 2003, 1, 13.
12. Masuchi, M. H.; Celeghini, R. M. S.; Gonçalves, L. A. G.; Grimaldi R.; Quim. Nova 2008, 31, 1057.

13. Serbinova, E. A.; Tsuchiya, M.; Goth, S.; Kagan, V. E.; Packer, L. Em ref. 4 , cap. 2.

14. Frankel, E. N.; Food Chem. 1996, 57, 51

15. Mutalib, M. S. A.; Khaza'ai, H.; Wahle, K. W. L.; Food Res. Int. 1992, 36,405 .

16. Eitenmiller, R. R.; Food Tech. 1997, 51, 78.

17. http://www.ars.usda.gov/Services/docs.htm?docid=17477, acessada em Setembro 2009.

18. Rupérez, F. J.; Martín, D.; Herrera, E.; Barbas, C.; J. Chromatogr., A 2001, 935,45 .

19. Simone, A. H.; Eitenmiller, R. R.; J. Agric. Food Chem. 1998, 46, 5273.

20. Barrera-Arellano, D.; Méndez, V. R.; Velasco, J.; Ruiz, G. M.; Dobarganes, C.; J. Sci. Food Agric. 2002, 82, 1696.

21. Del Caro, A.; Vacca, V.; Poiana, M.; Fenu, P.; Piga, A.; Food Chem. 2006, 98, 311.

22. Syväoja, E. L.; Piironen, V.; Varo, P.; Koivistoinen, P.; Salminen, K.; Milchwissenschaft 1985, 40, 467.

23. Kang, K. R.; Cherian, G.; Sim, J. S.; J. Food Sci. 1998, 63, 592.

24. IBGE; Pesquisa de orçamentos familiares 1995-1996 (POF): Consumo alimentar domiciliar per capita, Rio de Janeiro, 1998, vol. 3, p. 40.

25. Abidi, S. L.; J. Chromatogr., A 2000, 881, 197.

26. Abidi, S. L.; Rennick, K. A.; J. Chromatogr., A 2001, 913, 379.

27. Kamal-Eldin, A.; Görgen, S.; Petterson, J.; Lampi, A. M.; J. Chromatogr., A 2000, 881, 217.

28. Lee, J.; Kin, Y.; Landen, W. O.; Eitenmiller Jr., R. R.; J. Food Composit. Anal. 2000, 3, 457.

29. Shin, T.-S.; Godber, J. S.; J. Am. Oil Chem. Soc. 1993, 70, 1289

30. Rios, M. D. G.; Penteado, M. V. C.; Quim. Nova 2003, 26, 12.

31. Guinazi, M.; Dissertação de Mestrado, Universidade Federal de Viçosa, Brasil, 2004.

32. Syväoja, E.-L.; Piironen, V.; Varo, P.; Koivistoinen, P.; Salminen, K.; J. Am. Oil Chem. Soc. 1986, 63, 328.

33. Dionisi, F.; Prodolliet, J.; Tagliaferri, E.; J. Am. Oil Chem. Soc. 1995, 72,1505 .

34. Ryynänen, M.; Lampi, A.-M.; Salo-Vänänen, P.; Ollilainen, V.; Piironen, V.; J Food Composit. Anal. 2004, 17, 749.

35. Piironen, V.; Syväoja, E. L.; Varo, P.; Salminen, K.; Koivistoinen, P.; J. Agric.Food Chem. 1986, 34, 742.

36. Cert, A.; Moreda, W.; Camino, M. C. P.; J. Chromatogr., A 2000, 881, 131.

37. Bianchini-Pontuschka, R.; Penteado, M. V. C.; Vitaminas: aspectos nutricionais, bioquímicos, clínicos e analíticos, Manole: Barueri, 2003.

38. Ahmed, M. K.; Daun, J. D.; Przybylski, R.; J. Food Comp. Anal. 2005, 18,359 .

39. Rossi, M.; Alamprese, C.; Ratt,i S.; Food Chem. 2007, 102, 812.

40. Schwartz, H.; Ollilainen, V.; Piironen, V.; Lampi, A.; J. Food Comp. Anal. 2008, 21, 152

41. Vallilo, M. I.; Caruso, M. E. S.; Takemoto, E.; Pimentel, S.; Rev. Inst. Flor. 2007, 18, 73.

42. Takemoto, E.; Okada, I. A.; Garbelotti, M. L.; Tavares, M.; AuedPimentel, S.; Rev. Inst. Adolfo Lutz 2001, 60, 113.

43. Philippi, S. T.; Latterza, A . R.; Cruz, A. T. R.; Ribeiro, L. C.; Rev. Nut. 1999, 12,65 .

44. http://www.nap.edu/catalog.php?record_id=9810, acessada em Setembro 2009.

45. Trichopoulou, A.; Naska, A.; Vasilopoulou, E.; Int. J. Vitam. Nutr. Res. 2001, 71, 149 